\title{
4D models of de Sitter uplift
}

\author{
Renata Kallosh, ${ }^{1, *}$ Andrei Linde, ${ }^{1, \dagger}$ Evan McDonough, ${ }^{2, \hbar}$ and Marco Scalisi ${ }^{3, \S}$ \\ ${ }^{1}$ Stanford Institute for Theoretical Physics and Department of Physics, Stanford University, \\ Stanford, California 94305, USA \\ ${ }^{2}$ Department of Physics, Brown University, Providence, Rhode Island 02903, USA \\ ${ }^{3}$ Institute for Theoretical Physics, KU Leuven, Celestijnenlaan 200D, B-3001 Leuven, Belgium
}

(Received 2 October 2018; published 12 February 2019)

\begin{abstract}
It was shown in [1] that the modified 4D version of the Kachru, Kallosh, Linde, and Trivedi (KKLT) model proposed in [2] is inconsistent for large values of the parameter $c$ advocated in [2], since there is a point in the moduli space where $\left|D_{S} W\right|^{2}$ vanishes. The authors responded with yet another modification of the 4D KKLT model [3]. However, for large $c$, this model suffers from an even worse problem: not only is there a point in the moduli space where $\left|D_{S} W\right|^{2}$ vanishes, there is also a region in the moduli space where $\left|D_{S} W\right|^{2}$ is negative. Meanwhile, for small $c$ these models have de Sitter (dS) vacua. We construct improved models, which are fully consistent for all values of parameters, just as the original version of the KKLT model, using a nilpotent superfield. These models have a family of dS vacua for a broad range of parameter values. Thus, the results of the analysis of all presently available consistent generalizations of the 4D KKLT model, in the domain of their validity, confirm the existence of dS vacua in the KKLT scenario.
\end{abstract}

DOI: 10.1103/PhysRevD.99.046006

\section{INTRODUCTION}

The recent Kachru, Kallosh, Linde, and Trivedi (KKLT) debate between $[1,2]$ currently involves two sides of the story. One is based on the 10D analysis presented in [2], recently summarized in [3]. In the latter paper, the authors reviewed their previous long paper [2], presenting a short version of their arguments in 10D. In our opinion, expressed in [1], their results are based on several unjustified and debatable assumptions. In the absence of actual computations in [2], the status of the 10D arguments will remain inconclusive until such an explicit analysis is actually performed. A similar conclusion was reached in [4].

Meanwhile, in 4D the situation is more transparent, being based on 4D supergravity with a nilpotent multiplet $S$, representing an anti-D3-brane. Here the explicit equations can be easily checked. In [2] a Kähler potential $K$ and a superpotential $W$ were given, which were supposed to confirm the 10D analysis in [2]. The authors of [2] conceded in [3] that their first model (we will call it v1)

\footnotetext{
*kallosh@stanford.edu

alinde@stanford.edu

*evan_mcdonough@brown.edu

${ }^{\S}$ marco.scalisi@kuleuven.be
}

Published by the American Physical Society under the terms of the Creative Commons Attribution 4.0 International license. Further distribution of this work must maintain attribution to the author(s) and the published article's title, journal citation, and DOI. Funded by SCOAP . is inconsistent for the values of the parameters $|c A| \gg b$ advocated in [2].

Therefore, they have now proposed another version of their model in [3] (we will call it v2). We will study the model v2 and perform the corresponding analysis of $\left|D_{S} W\right|^{2}$ as a function of $T$. Surprisingly, we see again that $\left|D_{S} W\right|^{2}$ can vanish and even be negative, which invalidates the new model proposed in [3] for large $c$. Meanwhile, for small $c$ this model has a family of de Sitter (dS) vacua. This is in contradiction with the claim in [3] that their model v2 is "better" than their model $\mathrm{v} 1$, and "can match the ten dimensional result." On the contrary, we find that the second model, in the domain of its validity, supports the standard conclusion of the existence of dS vacua in the KKLT model.

Finally, we also suggest how to modify the models in $[2,3]$ so that the positivity of $\left|D_{S} W\right|^{2}$ is preserved for all values of their parameters. In these new models, we still find dS solutions. This means that, even when a model consistently deviates from the original KKLT scenario, metastable dS vacua are still preserved.

\section{MODELS V1 AND V2}

\section{A. Model v1}

The original version of the KKLT scenario in the formulation where the anti-D3-brane is represented via a nilpotent multiplet $S$ is given by [5-7]

$$
W=W_{0}+A e^{-a T}+b S
$$


and the Kähler potential which can be either

$$
K=-3 \log (T+\bar{T})+S \bar{S},
$$

or

$$
K=-3 \log (T+\bar{T}-S \bar{S}) .
$$

The modification proposed in [2] introduces an extra term $c A e^{-a T} S$ in the superpotential, with an extra parameter $c$, which is supposed to describe effects of backreaction

$$
W=W_{0}+A e^{-a T}+c A e^{-a T} S+b S .
$$

It was argued in [2] that $|c A| \gg b$. This argument, which is also central to their 10D approach, does not seem well motivated, because it would imply that the backreaction to the anti-D3-brane is much greater than the main effect of the anti-D3-brane [1,4]. We studied the general case, including $|c A| \ll b$ as well as $|c A| \gg b$.

After Kähler transformation

$$
\left(c A e^{-a T}+b\right) S \rightarrow \tilde{S}
$$

an equivalent model is (ignoring tilde)

$$
\begin{gathered}
W=W_{0}+A e^{-a T}+S, \\
K=-3 \log \left(T+\bar{T}-\frac{S \bar{S}}{\left|c A e^{-a T}+b\right|^{2}}\right) .
\end{gathered}
$$

Note that the denominator in (7) is a perfect square, $\left|c A e^{-a T}+b\right|^{2} \equiv\left(c A e^{-a T}+b\right)\left(c A e^{-a \bar{T}}+b\right)$. It is positive everywhere except the point

$$
T_{0}=\frac{1}{a} \ln \left(-\frac{c A}{b}\right)
$$

where it vanishes. This makes the use of the nilpotent multiplet $S$ in the model (4) inconsistent [1]. This violation of the consistency requirement occurs at large volume, e.g., $\left|T_{0}\right| \simeq 120$ for $\{a, A, b, c\}=\left\{0.1,1,10^{-5}, 1\right\}$, and hence is a problem in precisely the region of moduli space where the nilpotent multiplet is expected to provide a valid effective field theory description of an anti-D3-brane.

If one disregards this problem of the model proposed in [2] and calculates the resulting potential, one finds that the theory does contain a large family of $\mathrm{dS}$ vacua, some of which have not been found in the previous works [1]. The authors of [2] argued that we found dS states for $c=1$, whereas we found dS states for a very broad set of parameters, starting from very small $c$, all the way to $c=10^{4} \gg b$.

\section{B. Model v2}

Consider a new model v2 [2]. We are given the superpotential (6) and Kähler potential

$$
K=-3 \log \left(T+\bar{T}-\frac{S \bar{S}}{\tilde{b}^{2}}\right)
$$

with $T=t+i \theta$ and $\tilde{b}^{2}$ depending on some arbitrary functions $f(T+\bar{T})$ and $g(T+\bar{T})$, such as

$\tilde{b}^{2}=b^{2}+b\left(f(T+\bar{T}) e^{-a T}+\right.$ c.c. $)+g(T+\bar{T}) e^{-a(T+\bar{T})}$.

However, the model that was called better has a particular choice of these two functions, $f(T+\bar{T})=\bar{c}$ and $g(T+\bar{T})=g_{1} \cdot(T+\bar{T})$, where $g_{1}$ is a constant. So we proceed from there and define the v2 Kähler as follows. First, we reorganize the expression to take out the nilpotent field from the $\log$

$$
K=-3 \log \left((T+\bar{T})\left(1-\frac{S \bar{S}}{(T+\bar{T}) \tilde{b}^{2}}\right)\right)
$$

so that

$$
K=-3 \log (T+\bar{T})-3 \log \left(1-\frac{S \bar{S}}{(T+\bar{T}) \tilde{b}^{2}}\right),
$$

and, finally,

$$
K=-3 \log (T+\bar{T})+\frac{3}{(T+\bar{T}) \tilde{b}^{2}} S \bar{S} .
$$

Thus, we have

$K^{S \bar{S}}=\frac{T+\bar{T}}{3}\left(b^{2}+b\left(\bar{c} e^{-a T}+\right.\right.$ c.c. $\left.)+g_{1}(T+\bar{T}) e^{-a(T+\bar{T})}\right)$.

Thus, in order to disprove KKLT, the authors of [2] introduce a highly sophisticated modification of the original KKLT model [5-7], containing not one, but two extra parameters, $c$ and $g_{1}$.

We are now ready to compute the supersymmetry breaking in the $S$ direction, that is $\left|D_{S} W\right|^{2} \equiv D_{S} W K^{S \bar{S}} \bar{D}_{\bar{S}} \bar{W}$ and to study it at $S=0$. Note that the sign of $\left|D_{S} W\right|^{2}$ is determined by the sign of $K^{S \bar{S}}$. Since $D_{S} W=1$, we find

$$
\left|D_{S} W\right|^{2}=\frac{T+\bar{T}}{3}\left(b^{2}+b\left(\bar{c} e^{-a T}+\text { c.c }\right)+2 g_{1} t e^{-a(T+\bar{T})}\right) .
$$

In terms of $t, \theta$ we can present it as follows at $t>0$ and in the simple case $c=\bar{c}$,

$$
\left|D_{S} W\right|^{2}=\frac{2 t}{3}\left(b^{2}+2 b c e^{-a t} \cos a \theta+2 g_{1} t e^{-2 a t}\right) .
$$

If we take $\theta=\pi / a$, we find 


$$
\left|D_{S} W\right|^{2}=\frac{2 t}{3}\left(b^{2}-2 b c e^{-a t}+2 g_{1} t e^{-2 a t}\right) .
$$

As before, for positive $b c$ we find that the vanishing of $\left|D_{S} W\right|^{2}$ can be achieved in the complex plane of $T$ with positive $T+\bar{T}$. This makes the model $\mathrm{v} 2$ inconsistent to the same degree as model $v 1$, where the corresponding equation was

$$
\left|D_{S} W\right|^{2}=\frac{2 t}{3}\left|b+c e^{-a T}\right|^{2}>0 .
$$

Thus, if we take $\theta=\pi / a$ in (16), we find a point in field space where $\left|D_{S} W\right|^{2}=0$, which invalidates the model for $c>\frac{b}{2}$. A similar conclusion is reached for $|c|$ in the general case $c=|c| e^{i \gamma}$, but for a different value of $\theta$, depending on the phase $\gamma$.

In addition to a possibility that in model v2 $\left|D_{S} W\right|^{2}=0$ is possible, one finds that at small $t$, where the third term in Eq. (17) is small, the expression in (17) is negative, and the model is inconsistent for $c>\frac{b}{2}$. Meanwhile for $c<b / 2$ the corrections proportional to $c e^{-a T}$ are exponentially suppressed as compared to $b$. Therefore, the only relevant "backreaction" term is the one proportional to $g_{1}$. We checked that dS vacua exist in this model even if $g_{1} \gg b$, just as in the previous model (4).

\section{CONSISTENT GENERALIZATIONS OF THE KKLT MODEL}

As we have shown above, both versions of the modified KKLT construction proposed in $[2,3]$ are inconsistent for some values of their parameters, because both of them violate the consistency requirement for the description of the anti-D3-branes in terms of the nilpotent multiplet. Now we will solve this problem and propose some models that are consistent for all values of their parameters.

We will keep the original version of $W(6)$ and make a minor modification of the Kähler potential

$K=-3 \log \left(T+\bar{T}-\frac{S \bar{S}}{\left|c A e^{-a T}+b\right|^{2}+\beta c^{2} A^{2} e^{-a(T+\bar{T})}}\right)$,

where $\beta$ is some positive number. This immediately makes $K^{S \bar{S}}$ strictly positive definite, which avoids all inconsistencies of the models of $[2,3]$ for any choice of $\beta>0$. This model falls in the category of models previously studied in $[8,9]$.

Note that for $0<\beta \ll 1$, the KKLT potential in this model practically coincides with the potential obtained in our paper [1]. Thus all our previous results about the existence of dS vacua contained in [1] are confirmed for a large range of parameters, without any problems with the nilpotent multiplet $S$ encountered in $[2,3]$.

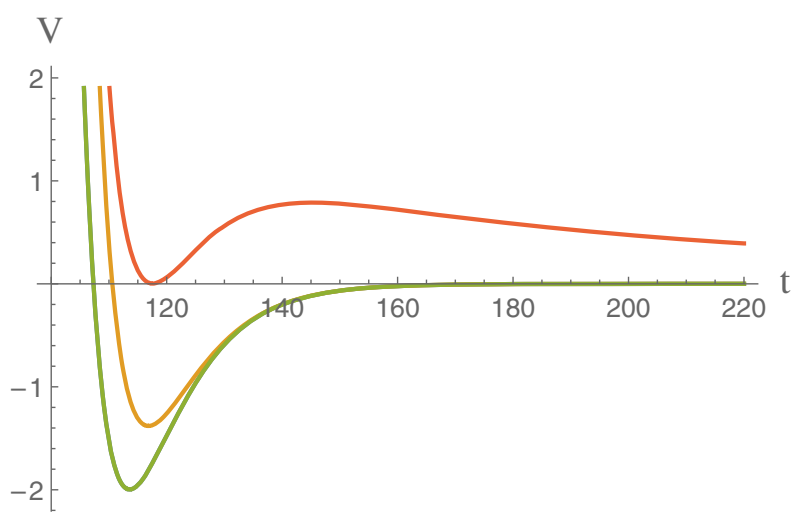

FIG. 1. The potential for the model (20) (multiplied by $10^{15}$ ) for $A=1, a=0.1, W_{0}=-10^{-4}, c=1$. The green (lower) line shows the potential with a supersymmetric anti-de Sitter minimum prior to uplifting, at $b=c=0$. The second (yellow) line shows the potential at $b=0$ uplifted by increase of $c$ to $c=1$. This does not uplift the potential to dS. Finally, the upper (red) line shows the potential with a dS (nearly Minkowski) minimum for $c=1, b=1.51 \times 10^{-5}$.

Yet another, even simpler, model is described by $W(6)$ and Kähler potential

$$
K=-3 \log \left(T+\bar{T}-\frac{S \bar{S}}{|b|^{2}+|c|^{2} e^{-a(T+\bar{T})}}\right),
$$

which amounts to the choice $f=0$ and $g=|c|^{2}$ in (10). $K^{S S}$ is strictly positive definite, which makes it consistent for any choice of $\{b, c\}$. This model also belongs to the class of models previously studied in $[8,9]$.

This model, just as the original KKLT model where the anti-D3-brane is represented via a nilpotent multiplet $S$ [5-7], has dS vacua for a broad choice of its parameters. We illustrate our results for the case $c=1, b=1.51 \times 10^{-5}$ in Fig. 1. It should be compared to Fig. 2 in our previous paper [1].

\section{CONCLUSION}

In this paper we have revisited the inconsistencies of the 4D supergravity analysis done in [2], found previously in [1] for large values of $c$ advocated in [2]. The authors of [2] responded to [1] with a revised model [3], which we have referred to as model v2. In this paper we have found the same, and yet further, inconsistencies in the revised model at $c>b / 2$ : (1) as in the original model, the supersymmetry breaking $\left|D_{S} W\right|^{2}=K^{S \bar{S}}$ vanishes at a point in moduli space, and (2) $K^{S \bar{S}}$ can in fact become negative. Such models do not have an embedding in de Sitter supergravity, at least as it is currently formulated, as is the case for the model given in [2].

However, with simple modifications, which we outline in Sec. III, these inconsistencies can be removed, leading to 
a family of dS solutions without problems 1 or 2 mentioned above. The results of the detailed analysis of various consistent generalizations of the 4D KKLT models, in the domain of their validity, invariably confirm the existence of $\mathrm{dS}$ vacua in the KKLT scenario.

\section{ACKNOWLEDGMENTS}

We would like to thank Shamit Kachru, Ander Retolaza, Eva Silverstein, Sandip Trivedi, Thomas Van Riet, and Timm Wrase for helpful comments and discussions. The work of
R. K. and A. L. is supported by Stanford Institute For Theoretical Physics (SITP), by the NSF Grant No. PHY1720397 and by the Simons Foundation grant. E. M. is supported in part by the National Science and Engineering Research Council of Canada via a PDF fellowship. M. S. is supported by the Research FoundationFlanders (FWO) and the European Union's Horizon 2020 research and innovation programme under the Marie Skłodowska-Curie Grant agreement No. 665501.
[1] R. Kallosh, A. Linde, E. McDonough, and M. Scalisi, de Sitter Vacua with a nilpotent superfield, Fortschr. Phys. 67, 1800068 (2019).

[2] J. Moritz, A. Retolaza, and A. Westphal, Toward de Sitter space from ten dimensions, Phys. Rev. D 97, 046010 (2018).

[3] J. Moritz, A. Retolaza, and A. Westphal, On uplifts by warped anti-D3-branes, arXiv:1809.06618.

[4] M. Cicoli, S. de Alwis, A. Maharana, F. Muia, and F. Quevedo, de Sitter vs quintessence in string theory, Fortschr. Phys. 67, 1800079 (2019).

[5] S. Ferrara, R. Kallosh, and A. Linde, Cosmology with nilpotent superfields, J. High Energy Phys. 10 (2014) 143.
[6] R. Kallosh and T. Wrase, Emergence of spontaneously broken supersymmetry on an anti-D3-brane in KKLT dS vacua, J. High Energy Phys. 12 (2014) 117.

[7] E. A. Bergshoeff, K. Dasgupta, R. Kallosh, A. Van Proeyen, and T. Wrase, $\overline{\mathrm{D} 3}$ and dS, J. High Energy Phys. 05 (2015) 058.

[8] E. McDonough and M. Scalisi, Inflation from nilpotent Kähler corrections, J. Cosmol. Astropart. Phys. 11 (2016) 028.

[9] R. Kallosh, A. Linde, D. Roest, and Y. Yamada, $\overline{D 3}$ induced geometric inflation, J. High Energy Phys. 07 (2017) 057. 